Research Article

\title{
On Asymptotic Pointwise Contractions in Modular Metric Spaces
}

\author{
Afrah A. N. Abdou \\ Department of Mathematics, King Abdulaziz University, P.O. Box 80203, Jeddah 21589, Saudi Arabia \\ Correspondence should be addressed to Afrah A. N. Abdou; fixedmathstar@hotmail.com
}

Received 20 April 2013; Accepted 17 May 2013

Academic Editor: Geraldo Botelho

Copyright (C) 2013 Afrah A. N. Abdou. This is an open access article distributed under the Creative Commons Attribution License, which permits unrestricted use, distribution, and reproduction in any medium, provided the original work is properly cited.

In this paper we study and prove some new fixed points theorems for pointwise and asymptotic pointwise contraction mappings in modular metric spaces.

\section{Introduction}

The notion of modulars on linear spaces and the corresponding theory of modular linear spaces were founded by Nakano [1] and were extensively developed by his mathematical school. In many cases, particularly in applications to integral operators, approximation and fixed point results, modular type conditions are much more natural as modular type assumptions can be more easily verified than their metric or norm counterparts. In present time the theory of modulars and modular spaces is extensively investigated. Chistyakov in $[2,3]$ introduced the notion of a Modular metric on arbitrary set and their corresponding modular spaces. The main idea behind this new concept according to Chistyakov is the physical interpretation of the modular. Informally speaking, whereas a metric on a set represents nonnegative finite distances between any two points of the set, a modular on a set attributes a nonnegative (possibly, infinite valued) "field of (generalized) velocities": to each "time" $\lambda>0$ (the absolute value of) an average velocity $w_{\lambda}(x, y)$ is associated in such a way that in order to cover the "distance" between points $x, y \in X$ it takes time $\lambda$ to move from $x$ to $y$ with velocity $w_{\lambda}(x, y)$. But our approach to modular metric spaces is different. Indeed we look at these spaces as the nonlinear version of the classical modular spaces as introduced by Nakano [1] on vector spaces and modular function spaces introduced by Musielak [4] and Orlicz [5].

In recent years, there was a strong interest to study the fixed point property in modular function spaces, which are natural generalization of both function and sequence spaces, after the first paper [6] was published by Khamsi et al. in 1990. More recently, the authors presented some fixed point results for pointwise contractions and asymptotic pointwise contractions acting in modular functions spaces $[7,8]$. The theory of contractions and nonexpansive mappings defined on convex subsets of Banach spaces has been well developed since the 1960s (see, e.g., Belluce and Kirk $[9,10]$, Browder [11], Bruck [12], DeMarr [13], and Lim [14]) and generalized to other metric spaces (see, e.g., [15-17]) and modular function spaces (see, e.g., [6]). The corresponding fixed point results were then extended to larger classes of mappings like pointwise contractions $[18,19]$ and asymptotic pointwise contractions and nonexpansive mappings [20,21].

In this paper we prove the existence of fixed point theorems for pointwise mappings without the use of ultrapower technique. Our results extend and improve several known results including the corresponding recent fixed point results of $[7,8,20]$.

For more on metric fixed point theory, the reader may consult the book [22].

\section{Basic Definitions and Properties}

Let $X$ be a nonempty set. Throughout this paper for a function $w:(0, \infty) \times X \times X \rightarrow(0, \infty]$, we will write

$$
w_{\lambda}(x, y)=w(\lambda, x, y) \text {, }
$$

for all $\lambda>0$ and $x, y \in X$. 
Definition 1 (see $[2,3])$. A function $w:(0, \infty) \times X \times X \rightarrow$ $[0, \infty]$ is said to be modular metric on $X$ if it satisfies the following axioms:

(i) $x=y$ if and only if $w_{\lambda}(x, y)=0$, for all $\lambda>0$;

(ii) $w_{\lambda}(x, y)=w_{\lambda}(y, x)$, for all $\lambda>0$, and $x, y \in M$;

(iii) $w_{\lambda+\mu}(x, y) \leq w_{\lambda}(x, z)+w_{\mu}(z, y)$, for all $\lambda, \mu>0$ and $x, y, z \in X$.

If instead of (i), we have only the condition $\left(i^{\prime}\right)$

$$
w_{\lambda}(x, x)=0, \quad \forall \lambda>0, x \in X
$$

then $w$ is said to be a pseudomodular (metric) on $X$. A modular metric $w$ on $X$ is said to be regular if the following weaker version of (i) is satisfied:

$$
x=y \quad \text { iff } w_{\lambda}(x, y)=0 \text {, for some } \lambda>0 \text {. }
$$

Finally $w$ is said to be convex if for $\lambda, \mu>0$ and $x, y, z \in X$, it satisfies the inequality

$$
w_{\lambda+\mu}(x, y) \leq \frac{\lambda}{\lambda+\mu} w_{\lambda}(x, z)+\frac{\mu}{\lambda+\mu} w_{\mu}(z, y) \text {. }
$$

Note that for a metric pseudomodular $w$ on a set $X$, and any $x, y \in X$, the function $\lambda \rightarrow w_{\lambda}(x, y)$ is nonincreasing on $(0, \infty)$. Indeed if $0<\mu<\lambda$, then

$$
w_{\lambda}(x, y) \leq w_{\lambda-\mu}(x, x)+w_{\mu}(x, y)=w_{\mu}(x, y) .
$$

Definition 2 (see $[2,3])$. Let $w$ be a pseudomodular on $X$. Fix $x_{0} \in X$. The two sets

$$
\begin{aligned}
X_{w} & =X_{w}\left(x_{0}\right)=\left\{x \in X: w_{\lambda}\left(x, x_{0}\right) \longrightarrow 0 \text { as } \lambda \longrightarrow \infty\right\}, \\
X_{w}^{*} & =X_{w}^{*}\left(x_{0}\right) \\
& =\left\{x \in X: \exists \lambda=\lambda(x)>0 \text { such that } w_{\lambda}\left(x, x_{0}\right)<\infty\right\}
\end{aligned}
$$

are said to be modular spaces (around $x_{0}$ ).

It is clear that $X_{w} \subset X_{w}^{*}$, but this inclusion may be proper in general. It follows from $[2,3]$ that if $w$ is a modular on $X$, then the modular space $X_{w}$ can be equipped with a (nontrivial) metric, generated by $w$ and given by

$$
d_{w}(x, y)=\inf \left\{\lambda>0: w_{\lambda}(x, y) \leq \lambda\right\}
$$

for any $x, y \in X_{w}$. If $w$ is convex modular on $X$, according to $[2,3]$ the two modular spaces coincide, $X_{w}^{*}=X_{w}$, and this common set can be endowed with the metric $d_{w}^{*}$ given by

$$
d_{w}^{*}(x, y)=\inf \left\{\lambda>0: w_{\lambda}(x, y) \leq 1\right\}
$$

for any $x, y \in X_{w}$. These distances will be called Luxemburg distances (see example below for the justification).
Next we give an example of a modular metric space.

Example 3. Let $\Omega$ be a nonempty set and $\Sigma$ be a nontrivial $\sigma$-algebra of subsets of $\Omega$. Let $\mathscr{P}$ be a $\delta$-ring of subsets of $\Omega$, such that $E \cap A \in \mathscr{P}$ for any $E \in \mathscr{P}$ and $A \in \Sigma$. Let us assume that there exists an increasing sequence of sets $K_{n} \in$ $\mathscr{P}$ such that $\Omega=\bigcup K_{n}$. By $\mathscr{E}$ we denote the linear space of all simple functions with supports from $\mathscr{P}$. By $\mathscr{M}_{\infty}$ we will denote the space of all extended measurable functions, that is all functions $f: \Omega \rightarrow[-\infty, \infty]$ such that there exists a sequence $\left\{g_{n}\right\} \subset \mathscr{E},\left|g_{n}\right| \leq|f|$ and $g_{n}(\omega) \rightarrow f(\omega)$ for all $\omega \in \Omega$. By $1_{A}$ we denote the characteristic function of the set $A$. Let $\rho: \mathscr{M}_{\infty} \rightarrow[0, \infty]$ be a nontrivial, convex, and even function. We say that $\rho$ is a regular convex function pseudomodular if

(i) $\rho(0)=0$;

(ii) $\rho$ is monotone; that is, $|f(\omega)| \leq|g(\omega)|$ for all $\omega \in \Omega$ implies $\rho(f) \leq \rho(g)$, where $f, g \in \mathscr{M}_{\infty}$;

(iii) $\rho$ is orthogonally subadditive; that is, $\rho\left(f 1_{A \cup B}\right) \leq$ $\rho\left(f 1_{A}\right)+\rho\left(f 1_{B}\right)$ for any $A, B \in \Sigma$ such that $A \cap$ $B \neq \emptyset, f \in \mathscr{M}$;

(iv) $\rho$ has the Fatou property; that is, $\left|f_{n}(\omega)\right| \uparrow|f(\omega)|$ for all $\omega \in \Omega$ implies $\rho\left(f_{n}\right) \uparrow \rho(f)$, where $f \in \mathscr{M}_{\infty}$;

(v) $\rho$ is order continuous in $\mathscr{E}$; that is, $g_{n} \in \mathscr{E}$ and $\left|g_{n}(\omega)\right| \downarrow 0$ implies $\rho\left(g_{n}\right) \downarrow 0$.

Similarly as in the case of measure spaces, we say that a set $A \in \Sigma$ is $\rho$-null if $\rho\left(g 1_{A}\right)=0$ for every $g \in \mathscr{E}$. We say that a property holds $\rho$-almost everywhere if the exceptional set is $\rho$-null. As usual we identify any pair of measurable sets whose symmetric difference is $\rho$-null as well as any pair of measurable functions differing only on a $\rho$-null set. With this in mind we define

$$
\mathscr{M}(\Omega, \Sigma, \mathscr{P}, \rho)=\left\{f \in \mathscr{M}_{\infty} ;|f(\omega)|<\infty \quad \rho-\text { a.e }\right\},
$$

where each $f \in \mathscr{M}(\Omega, \Sigma, \mathscr{P}, \rho)$ is actually an equivalence class of functions equal $\rho$-a.e. rather than an individual function. Where no confusion exists we will write $\mathscr{M}$ instead of $\mathscr{M}(\Omega$, $\Sigma, \mathscr{P}, \rho)$. Let $\rho$ be a regular function pseudomodular.

(a) We say that $\rho$ is a regular function semimodular if $\rho(\alpha f)=0$ for every $\alpha>0$ implies $f=0 \quad \rho$-a.e.

(b) We say that $\rho$ is a regular function modular if $\rho(f)=0$ implies $f=0 \quad \rho$-a.e.

The class of all nonzero regular convex function modulars defined on $\Omega$ will be denoted by $\Re$. Let us denote $\rho(f, E)=$ $\rho\left(f 1_{E}\right)$ for $f \in \mathscr{M}, E \in \Sigma$. It is easy to prove that $\rho(f, E)$ is a function pseudomodular in the sense of Definition 2.1.1 in [23] (more precisely, it is a function pseudomodular with the Fatou property). Therefore, we can use all results of the standard theory of modular function spaces as per the framework defined by Kozlowski in [23-25], and see also Musielak [4] for the basics of the general modular theory. Let $\rho$ be a convex function modular. 
(a) The associated modular function space is the vector space $L_{\rho}(\Omega, \Sigma)$, or briefly $L_{\rho}$, defined by

$$
L_{\rho}=\{f \in \mathscr{M} ; \rho(\lambda f) \longrightarrow 0 \text { as } \lambda \longrightarrow 0\}
$$

(b) The following formula defines a norm in $L_{\rho}$ (frequently called Luxemburg norm):

$$
\|f\|_{\rho}=\inf \left\{\alpha>0 ; \rho\left(\frac{f}{\alpha}\right) \leq 1\right\} .
$$

Modular function space furnishes a wonderful example of a modular metric space. Indeed let $L_{\rho}$ be modular function space. Define the function $w$ by

$$
w_{\lambda}(f, g)=\rho\left(\frac{f-g}{\lambda}\right) \text {, }
$$

for all $\lambda>0$, and $f, g \in L_{\rho}$; then $w$ is a modular metric on $L_{\rho}$. Note that $w$ is convex if and only if $\rho$ is convex. Moreover we have

$$
\|f-g\|_{\rho}=d_{w}^{*}(f, g)
$$

for any $f, g \in L_{\rho}$.

Definition 4. Let $X_{w}$ be a modular metric space.

(1) The sequence $\left(x_{n}\right)_{n \in \mathbb{N}}$ in $X_{w}$ is said to be $w$-convergent to $x \in X_{w}$ if and only if $w_{1}\left(x_{n}, x\right) \rightarrow 0$, as $n \rightarrow \infty$. $x$ will be called the $w$-limit of $\left(x_{n}\right)$.

(2) The sequence $\left(x_{n}\right)_{n \in N}$ in $X_{w}$ is said to be $w$-Cauchy if $w_{1}\left(x_{m}, x_{n}\right) \rightarrow 0$, as $m, n \rightarrow \infty$.

(3) A subset $M$ of $X_{w}$ is said to be $w$-closed if the $w$-limit of a $w$-convergent sequence of $M$ always belong to $M$.

(4) A subset $M$ of $X_{w}$ is said to be $w$-complete if any $w$ Cauchy sequence in $M$ is a $w$-convergent sequence and its $w$-limit is in $M$.

(5) A subset $M$ of $X_{w}$ is said to be $w$-bounded one has

$$
\delta_{w}(M)=\sup \left\{w_{1}(x, y) ; x, y \in M\right\}<\infty
$$

Note that if $w$ is regular, then the $w$-limit of a sequence is unique. In general if $\lim _{n \rightarrow \infty} w_{\lambda}\left(x_{n}, x\right)=0$, for some $\lambda>0$, then we may not have $\lim _{n \rightarrow \infty} w_{\lambda}\left(x_{n}, x\right)=0$, for all $\lambda>$ 0 . Therefore, as it is done in modular function spaces, we will say that $w$ satisfies $\Delta_{2}$-condition if this is the case; that is, $\lim _{n \rightarrow \infty} w_{\lambda}\left(x_{n}, x\right)=0$, for some $\lambda>0$ implies $\lim _{n \rightarrow \infty} w_{\lambda}\left(x_{n}, x\right)=0$, for all $\lambda>0$. In $[2,3]$, one will find a discussion about the connection between $w$-convergence and metric convergence with respect to the Luxemburg distances. In particular, we have

$$
\lim _{n \rightarrow \infty} d_{w}\left(x_{n}, x\right)=0 \quad \text { iff } \lim _{n \rightarrow \infty} w_{\lambda}\left(x_{n}, x\right)=0, \forall \lambda>0,
$$

for any $\left\{x_{n}\right\} \in X_{w}$ and $x \in X_{w}$. And in particular we have $w$-convergence and $d_{w}$ convergence are equivalent if and only if the modular $w$ satisfies the $\Delta_{2}$-condition. Moreover if the modular $w$ is convex, then we know that $d_{w}^{*}$ and $d_{w}$ are equivalent which implies

$$
\lim _{n \rightarrow \infty} d_{w}^{*}\left(x_{n}, x\right)=0 \text { iff } \lim _{n \rightarrow \infty} w_{\lambda}\left(x_{n}, x\right)=0, \forall \lambda>0,
$$

for any $\left\{x_{n}\right\} \in X_{w}$ and $x \in X_{w}[2,3]$.

Let $(X, w)$ be a modular metric space. Throughout the rest of this work, we will assume that $w$ satisfies the Fatou property; that is, if

$$
\lim _{n \rightarrow \infty} w_{1}\left(x_{n}, x\right)=0, \quad \lim _{n \rightarrow \infty} w_{1}\left(y_{n}, y\right)=0,
$$

then we must have

$$
w_{1}(x, y) \leq \liminf _{n \rightarrow \infty} w_{1}\left(x_{n}, y_{n}\right) .
$$

For any $x \in X_{w}$ and $r \geq 0$, we define the modular ball

$$
B_{w}(x, r)=\left\{y \in X_{w} ; w_{1}(x, y) \leq r\right\} .
$$

Note that if $w$ satisfies the Fatou property, then modular balls are $w$-closed. An admissible subset of $X_{w}$ is defined as an intersection of modular balls. Denote by $\mathscr{A}_{w}\left(X_{w}\right)$ the family of admissible subsets of $X_{w}$. Note that $\mathscr{A}_{w}\left(X_{w}\right)$ is stable by intersection. At this point we introduce some notation which will be used throughout the remainder of this work. For a subset $A$ of a modular metric space $X_{w}$ set

$$
\operatorname{cov}_{w}(A)=\bigcap\{B: B \text { is a modular ball and } A \subset B\} .
$$

Recall that $A$ is $w$-bounded if $\delta_{w}(A)=\sup \left\{w_{1}(x, y) ; x, y \in\right.$ $A\}<\infty$.

Definition 5. Let $(X, w)$ be a modular metric space. One will say that $\mathscr{A}_{w}\left(X_{w}\right)$ is compact if any family $\left(A_{\alpha}\right)_{\alpha \in \Gamma}$ of elements of $\mathscr{A}_{w}\left(X_{w}\right)$ has a nonempty intersection provided $\bigcap_{\alpha \in F} A_{\alpha} \neq \emptyset$ for any finite subset $F \subset \Gamma$.

Remark 6. Note that if $\mathscr{A}_{w}\left(X_{w}\right)$ is compact, then $X_{w}$ is $w$ complete.

Definition 7. Let $M$ be a modular metric space. A function $\Phi: M \rightarrow[0, \infty)$ is said to be

(i) $w$-convex if

$$
\{x ; \Phi(x) \leq r\} \in \mathscr{A}_{w}(M) \quad \text { for any } r \geq 0
$$

(ii) a type if it is defined as

$$
\Phi(u)=\limsup _{n \rightarrow \infty} w_{1}\left(x_{n}, u\right)
$$

where $\left(x_{n}\right)$ is a $w$-bounded sequence in $M$.

Types are very useful in the study of the geometry of Banach spaces and the existence of fixed point of mappings. We will say that $M$ is type-stable if types are $w$-convex. We have the following lemma. 
Lemma 8. Let $M$ be a modular metric space such that $\mathscr{A}_{w}(M)$ is compact on $M$ which is type-stable. Then for any type $\Phi$, there exists $z \in M$ such that

$$
\Phi(z)=\inf \{\Phi(x) ; x \in M\}
$$

The proof is easy and will be omitted.

\section{Pointwise Contraction in Modular Metric Spaces}

In $[2,3]$ the author defined Lipschitzian mappings in modular metric spaces and proved some fixed point theorems. In this paper, we propose a more general definition. Indeed, in the case of modular function spaces, it is proved in [6] that

$$
w_{\lambda}(T(x), T(y)) \leq w_{\lambda}(x, y), \quad \text { for any } \lambda>0
$$

if and only if

$$
d_{w}(T(x), T(y)) \leq d_{w}(x, y) .
$$

Moreover an example is given such that

$$
w_{1}(T(x), T(y)) \leq w_{1}(x, y),
$$

but $T$ is not Lipschitzian with respect to $d_{w}$ with constant 1 .

Definition 9. Let $(X, w)$ be a modular metric space. Let $M$ be a nonempty subset of $X_{w}$. A map $T: M \rightarrow M$ is called

(i) generalized contraction if there is an increasing and upper semicontinuous function $\psi: \mathbb{R}^{+} \rightarrow[0, \infty)$ satisfying $\psi(t)<t$, for $t>0$, such that for any $x, y \in$ M

$$
w_{1}(T(x), T(y)) \leq \psi\left(w_{1}(x, y)\right)
$$

(ii) generalized pointwise contraction if there is an increasing and upper semicontinuous function with respect to the second variable $\psi: M \times \mathbb{R}^{+} \rightarrow[0, \infty)$ satisfying $\psi(x, t)<t$, for $t>0$ and $x \in M$, such that

$$
w_{1}(T(x), T(y)) \leq \psi\left(x, w_{1}(x, y)\right),
$$

for any $x, y \in M$.

Now, we are ready to prove an analogue to Boyd and Wong fixed point theorem [26] in modular metric spaces.

Theorem 10. Let $(X, w)$ be a modular metric space. Let $M$ be a nonempty $w$-closed $w$-bounded subset of $X_{w}$. Assume that the family $\mathscr{A}_{w}(M)$ is compact and $T: M \rightarrow M$ is a generalized contraction. Then $T$ has a unique fixed point $z$. Moreover the orbit $\left\{T^{n}(x)\right\}$ converges to $z$, for each $x \in M$.

Proof. Let $x \in M$, we define the $w$-type

$$
\Phi(u)=\limsup _{n \rightarrow \infty} w_{1}\left(T^{n}(x), u\right), \quad \text { for each } u \in M \text {. }
$$

Since $\mathscr{A}_{w}(M)$ is compact, then for any $x \in M$, we have

$$
\Omega(x)=\bigcap_{n \geq 1} \operatorname{cov}_{w}\left(\left\{T^{k}(x) ; k \geq n\right\}\right) \neq \emptyset .
$$

Since $T$ is a generalized contraction, for any $x, y \in M$, we have

$$
w_{1}\left(T^{n}(x), T^{n}(y)\right) \leq \psi\left(w_{1}\left(T^{n-1}(x), T^{n-1}(y)\right)\right),
$$

which shows that $\left\{w_{1}\left(T^{n}(x), T^{n}(y)\right)\right\}$ is decreasing and bounded below. Therefore $w_{1}\left(T^{n}(x), T^{n}(y)\right)$ converges to $L \geq$ 0 . Thus we get $L \leq \psi(L)$ since $\psi$ is upper semicontinuous. Our assumptions on $\psi$ force $L=0$, which means that

$$
\lim _{n \rightarrow \infty} w_{1}\left(T^{n}(x), T^{n}(y)\right)=0 \quad \text { for any } x, y \in M \text {. }
$$

Now let $z$ be a fixed point of $T$; that is, $T(z)=z$, and then we have for any $x \in M$

$$
\lim _{n \rightarrow \infty} w_{1}\left(T^{n}(x), T^{n}(z)\right)=0 .
$$

Since $T^{n}(z)=z$, then $\lim _{n \rightarrow \infty} w_{1}\left(T^{n}(x), z\right)=0$; that is, if $T$ has a fixed point $z$, then any orbit $\left\{T^{n}(x)\right\} w$-converges to $z$. Note that if $u$ is another fixed point of $T$, then $\lim _{n \rightarrow \infty} w_{1}\left(T^{n}(u), z\right)=0$, so $w_{1}(u, z)=0$; that is, $u=v$. Therefore $T$ has at most one fixed point. Let us finish the proof of the theorem by showing that $T$ has a fixed point. Fix $x \in M$. Let $z \in \Omega(x)$. We have

$$
\Phi(z)=\limsup _{n \rightarrow \infty} w_{1}\left(T^{n}(x), z\right) \leq \delta_{w}(M)<\infty .
$$

Let $\varepsilon>0$. There exists $n_{0} \geq 1$, such that for every $n \geq n_{0}$,

$$
w_{1}\left(T^{n}(x), z\right) \leq \Phi(z)+\varepsilon .
$$

Now, for $n \geq n_{0}, h \geq 1$, and since $\psi$ is increasing, we get

$$
\begin{aligned}
w_{1}\left(T^{n+h}(x), T^{n}(z)\right) & \leq \psi^{h}\left(w_{1}\left(T^{n}(x), z\right)\right) \\
& \leq \psi^{h}(\Phi(z)+\varepsilon) .
\end{aligned}
$$

In particular, we have $T^{n+h}(x) \in B_{w}\left(T^{h}(z), \psi^{h}(\Phi(z)+\varepsilon)\right)$, for any $h \geq n_{0}$. So

$$
\begin{aligned}
\Omega(x) & \subset \operatorname{cov}_{w}\left(\left\{T^{n+h}(x) ; n \geq n_{0}\right\}\right) \\
& \subset B_{w}\left(T^{h}(z), \psi^{h}(\Phi(z)+\varepsilon)\right) .
\end{aligned}
$$

Since $z \in \Omega\left(T^{n}(x)\right)$, we get

$$
w_{1}\left(z, T^{h}(z)\right) \leq \psi^{h}(\Phi(z)+\varepsilon) .
$$

Since $\psi$ is upper semicontinuous, if we let $\varepsilon$ go to 0 , we get

$$
w_{1}\left(z, T^{h}(z)\right) \leq \psi^{h}(\Phi(z)), \quad \text { for any } h \geq 1 .
$$

Note that $\lim _{h \rightarrow \infty} \psi^{h}(\Phi(z))=0$. Indeed we have $\psi^{h}(\Phi(z)) \leq$ $\psi^{h-1}(\Phi(z))$; that is, $\left\{\psi^{h}(\Phi(z))\right\}$ is decreasing. Since $\Phi$ is a positive function, the sequence $\left\{\psi^{h}(\Phi(z))\right\}$ converges to some 
$L \geq 0$. Since $\psi$ is upper semicontinuous, we get $\psi(L) \leq L$. Our assumptions on $\psi$ will imply $L=0$. Therefore, we have

$$
\lim _{n \rightarrow \infty} w_{1}\left(z, T^{h}(z)\right)=0 .
$$

Similarly, we have $w_{1}\left(T(z), T^{h+1}(z)\right) \leq \psi\left(w_{1}\left(z, T^{h}(z)\right)\right)$, thus

$$
\lim _{n \rightarrow \infty} w_{1}\left(T(z), T^{h+1}(z)\right)=0 .
$$

Since $w$ is regular, the uniqueness of $w$-limit implies

$$
T(z)=z
$$

which prove that $z$ is a fixed point by $T$. In particular $\Omega(x)$ is reduced to one point.

Before we state our next result, we will need to define the concept of Chebyshev center and radius in modular metric spaces. Let $(X, w)$ be a modular metric space and let $A \subset X$ be a nonempty $w$-bounded subset. For any $x \in A$, define

$$
r_{x}(A)=\sup \left\{w_{1}(x, y) ; y \in A\right\} .
$$

The Chebyshev radius of $A$ is defined by

$$
R_{w}(A)=\inf \left\{r_{x}(A) ; x \in A\right\} .
$$

Obviously we have $R_{w}(A) \leq r_{x}(A) \leq \delta_{w}(A)$, for any $x \in A$. The Chebyshev center of $A$ is defined as

$$
C_{w}(A)=\left\{x \in A ; r_{x}(A)=R_{w}(A)\right\} .
$$

The following result is a generalization of Kirk's fixed point theorem [18] on pointwise contraction mappings.

Theorem 11. Let $(X, w)$ be a modular metric space. Let $M$ be a nonempty $w$-closed $w$-bounded subset of $X_{w}$. Assume that the family $\mathscr{A}_{w}(M)$ is compact and $T: M \rightarrow M$ is a generalized pointwise contraction. Then $T$ has a unique fixed point $z$. Moreover the orbit $\left\{T^{n}(x)\right\}$ converges to $z$, for each $x \in M$.

Proof. Since $\mathscr{A}_{w}(M)$ is compact, there exists a minimal nonempty $K \in \mathscr{A}_{w}(M)$ such that $T(K) \subset K$. It is easy to check that $\operatorname{cov}_{w}(T(K))=K$. Let us prove that $\delta_{w}(K)=0$; that is, $K$ is reduced to one point. Indeed since $M$ is $w$-bounded, then $\delta_{w}(K)<\infty$; that is, $K$ is also $w$-bounded. Let $z \in K$; then we have $K \subset B_{w}\left(z, r_{z}(K)\right)$. Since $T$ is a generalized pointwise contraction, there exists an increasing upper semicontinuous mapping with respect to the second variable $\psi: M \times \mathbb{R}^{+} \rightarrow$ $[0, \infty)$ such that

$$
w_{1}(T(x), T(y)) \leq \psi\left(x, w_{1}(x, y)\right) \text { for any } x, y \in M .
$$

In particular, we have $T(K) \subset B_{w}\left(T(x), \psi\left(x, r_{x}(K)\right)\right.$, for any $x \in K$, which implies

$$
\operatorname{cov}_{w}(T(K)) \subset B_{w}\left(T(x), \psi\left(x, r_{x}(K)\right) .\right.
$$

So, $K \subset B_{w}\left(T(x), \psi\left(x, r_{x}(K)\right)\right.$, which implies

$$
r_{T(x)}(K) \leq \psi\left(x, r_{x}(K)\right) \quad \text { for any } x \in K \text {. }
$$

This will force $\delta_{w}(K)=0$. Indeed fix $z \in K$ and define

$$
K_{z}=\left\{x \in K ; r_{x}(K) \leq r_{z}(K)\right\}
$$

Clearly $K_{z}$ is not empty since $z \in K_{z}$. Moreover we have

$$
K_{z}=\bigcap_{x \in K} B_{w}\left(x, r_{z}(K)\right) \cap K \in \mathscr{A}_{w}(M) .
$$

Since $r_{T(x)}(K) \leq \psi\left(x, r_{x}(K)\right) \leq r_{x}(K)$ for any $x \in K$, we get $T\left(K_{z}\right) \subset K_{z}$. The minimality behavior of $K$ implies $K_{z}=K$. In particular we have $r_{x}(K)=r_{z}(K)$ for any $x \in K$. Hence $\delta_{w}(K)=\sup _{x \in k} r_{x}(K)=r_{z}(K)$, for any $z \in K$. Since $r_{x}(K) \leq$ $\psi\left(x, r_{x}(K)\right)$, for any $x \in K$, we get

$$
\delta_{w}(K) \leq \psi\left(z, \delta_{w}(K)\right) .
$$

Assume $\delta_{w}(K) \neq 0$, then $\delta_{w}(K) \leq \psi\left(z, \psi\left(x, \delta_{w}(K)\right)<\delta_{w}(K)\right.$, which gives a contradiction. Thus $\delta_{w}(K)=0$; that is, $K$ is reduced to one point which is a fixed point by $T$ since $K$ is $T$-invariant. Hence $T$ has a fixed point. Next we prove that $T$ has a unique fixed point. Let $z$ and $u$ be two fixed points of $T$. We have

$$
w_{1}(z, u)=w_{1}(T(z), T(u)) \leq \psi\left(z, w_{1}(z, u)\right)
$$

Our assumptions on $\psi$ will then imply $w_{1}(z, u)=0$, that is, $z=u$. Next we finish the proof of our theorem by showing that, for any $x \in M$, the orbit $\left\{T^{n}(x)\right\} w$-converges to a fixed point of $T$. Indeed for any $x \in M$, we have

$$
w_{1}\left(z, T^{n}(x)\right) \leq \psi\left(z, w_{1}\left(z, T^{n-1}(x)\right)<w_{1}\left(z, T^{n-1}(x)\right) ;\right.
$$

that is, $\left\{w_{1}\left(z, T^{n}(x)\right)\right\}$ is decreasing. Let $L=\lim _{n \rightarrow \infty} w_{1} \times$ $\left(z, T^{n}(x)\right)$. Suppose that $L \neq 0$. Since $\psi$ is upper semicontinuous with respect to the second variable, we get

$$
L \leq \psi(z, L)<L
$$

which is a contradiction. Thus $\lim _{n \rightarrow \infty} w_{1}\left(z, T^{n}(x)\right)=0$; that is, the orbit $\left\{T^{n}(x)\right\} w$-converges to the fixed point $z$.

\section{Asymptotically Pointwise Contraction in Modular Metric Spaces}

Definition 12. Let $(X, w)$ be a modular metric space. Let $M$ be a nonempty subset of $X_{w}$. A mapping $T: M \rightarrow M$ is called an asymptotic pointwise Lipschitzian mapping if there exists a sequence of mappings $\alpha_{n}: M \rightarrow[0, \infty)$ such that

$$
w_{1}\left(T^{n}(x), T^{n}(y)\right) \leq \alpha_{n}(x) w_{1}(x, y) \quad \text { for any } x, y \in M \text {. }
$$

(i) If $\left\{\alpha_{n}\right\}$ converges pointwise to $\alpha: M \rightarrow[0,1)$, then $T$ is called an asymptotic pointwise contraction.

(ii) If $\limsup _{n \rightarrow \infty} \alpha_{n}(x) \leq k$, with $0<k<1$, then $T$ is called strongly asymptotic pointwise contraction. 
Note that if $T$ is asymptotic pointwise contraction, then $T$ has at most one fixed point. Indeed let $z$ and $u$ be two fixed points of $T$. Then

$$
w_{1}(z, u)=w_{1}\left(T^{n}(z), T^{n}(u)\right) \leq \alpha_{n}(z) w_{1}(z, u) .
$$

Since $\lim \sup _{n \rightarrow \infty} \alpha_{n}(z)<1$, then we must have $w_{1}(z, u)=0$; that is, $z=u$. A similar conclusion holds if $T$ is a strongly asymptotic pointwise contraction.

Theorem 13. Let $(X, w)$ be a modular metric space. Let $M$ be a nonempty $w$-closed $w$-bounded subset of $X_{w}$. Assume that the family $\mathscr{A}_{w}(M)$ is compact and $T: M \rightarrow M$ is a strongly asymptotic pointwise contraction. Then $T$ has a unique fixed point $z$. Moreover the orbit $\left\{T^{n}(x)\right\}$ converges to $z$, for each $x \in$ M.

Proof. Let $x \in M$ and define the type

$$
\Phi(a)=\limsup _{n \rightarrow \infty} w_{1}\left(T^{n}(x), a\right)
$$

for any $a \in M$. Since $\mathscr{A}_{w}(M)$ is compact, then

$$
\Omega(x)=\bigcap_{n \geq 1} \operatorname{cov}_{w}\left(\left\{T^{k}(x) ; k \geq n\right\}\right) \neq \emptyset .
$$

For any $n, m, h \geq 1$, we have

$$
\begin{aligned}
& w_{1}\left(T^{m+n+h}(x), T^{m+h}(x)\right) \\
& \quad \leq \alpha_{h}\left(T^{m}(x)\right) w_{1}\left(T^{n}(x), T^{m}(x)\right) .
\end{aligned}
$$

If we let $n$ go to infinity, we get

$$
\Phi\left(T^{m+h}(x)\right) \leq \alpha_{h}\left(T^{m}(x)\right) \Phi\left(T^{m}(x)\right) .
$$

Next we let $h$ go to infinity to get

$$
\limsup _{n \rightarrow \infty} \Phi\left(T^{n}(x)\right) \leq k \Phi\left(T^{m}(x)\right)
$$

for some $k \in(0,1)$, which easily implies that $\limsup _{n \rightarrow \infty} \Phi \times$ $\left(T^{n}(x)\right)=0$. Fix $z \in \Omega(x)$ and notice that

$$
\Phi(z) \leq \limsup _{n \rightarrow \infty} \Phi\left(T^{n}(x)\right) .
$$

Indeed let $a \in M$; then for any $\varepsilon>0$, there exists $n_{0} \geq 1$ such that for any $n \geq n_{0}$, we have

$$
w_{1}\left(T^{n}(x), a\right) \leq \Phi(a)+\varepsilon .
$$

In particular we have $T^{n}(x) \in B_{w}(a, \Phi(a)+\varepsilon)$, for any $n \geq n_{0}$. So

$$
\Omega(x) \subset \operatorname{cov}_{w}\left(\left\{T^{n}(x) ; n \geq n_{0}\right\}\right) \subset B_{w}(a, \Phi(a)+\varepsilon),
$$

which implies $z \in B_{w}(a, \Phi(a)+\varepsilon)$. This is true for any $\varepsilon>0$. Hence for any $a \in M$ we have $w_{1}(z, a) \leq \Phi(a)$. Therefore

$$
\Phi(z)=\limsup _{n \rightarrow \infty} w_{1}\left(T^{n}(x), z\right) \leq \limsup _{n \rightarrow \infty} \Phi\left(T^{n}(x)\right) .
$$

Therefore we have $\Phi(z)=0$; that is, $\left\{T^{n}(x)\right\}$ w-converges to $z$. This will force $z$ to be a fixed point of $T$. Indeed we have

$$
w_{2}(z, T(z)) \leq w_{1}\left(z, T^{n}(x)\right)+w_{1}\left(T(z), T^{n}(x)\right),
$$

which implies

$$
w_{2}(z, T(z)) \leq w_{1}\left(z, T^{n}(x)\right)+\alpha_{1}(z) w_{1}\left(z, T^{n-1}(x)\right),
$$

for any $n \geq 1$. Since $\left\{T^{n}(x)\right\} w$-converges to $z$, we get $w_{2}(z, T(z))=0$; that is, $T(z)=z$, since $w$ is regular. Since $T$ has at most one fixed point, we conclude that any orbit of $T w$-converges to the only fixed point $z$ of $T$.

Next we relax the strong behavior of $T$ but assume that types are convex to obtain the following result.

Theorem 14. Let $(X, w)$ be a modular metric space. Let $M$ be a nonempty $w$-closed $w$-bounded subset of $X_{w}$. Assume that the family $\mathscr{A}_{w}(M)$ is compact. Assume that $\mathscr{A}_{w}(M)$ is compact and type-stable. Let $T: M \rightarrow M$ be an asymptotic pointwise contraction. Then $T$ has a unique fixed point $z$. Moreover the orbit $\left\{T^{n}(x)\right\}$ converges to $z$, for each $x \in M$.

Proof. Similarly one can easily show that $T$ has at most one fixed point. As we did in the proof of the previous result, let $x \in M$ and define the type

$$
\Phi(a)=\limsup _{n \rightarrow \infty} w_{1}\left(T^{n}(x), a\right), \quad \text { for each } a \in M .
$$

Since $\mathscr{A}_{w}(M)$ is compact and type-stable, then there exists $z \in M$ such that

$$
\Phi(z)=\inf \{\Phi(a) ; a \in M\} .
$$

Let us show that $\Phi(z)=0$. Indeed we have

$$
w_{1}\left(T^{n+m}(x), T^{m}(z)\right) \leq \alpha_{m}(z) w_{1}\left(T^{n}(x), z\right),
$$

for any $n, m \geq 1$. If we let $n$ go to infinity, we get

$$
\Phi\left(T^{m}(z)\right) \leq \alpha_{m}(z) \Phi(z)
$$

which implies

$$
\Phi(z)=\inf \{\Phi(a) ; a \in M\} \leq \Phi\left(T^{m}(z)\right) \leq \alpha_{m}(z) \Phi(z) .
$$

If we let $m$ go to infinity, we get $\Phi(z) \leq \alpha(z) \Phi(z)$. Since $\alpha(z)<$ 1 , we get $\Phi(z)=0$, which implies that $\left\{T^{n}(x)\right\} w$-converges to $z$. This will force $z$ to be a fixed point of $T$. Since we already noticed that $T$ has at most one fixed point, then $T$ has a fixed point $z$ and any orbit $w$-converges to $z$.

\section{Acknowledgment}

The author would like to thank Professor M. A. Khamsi with whom the author had many fruitful discussions regarding this work. 


\section{References}

[1] H. Nakano, Modulared Semi-Ordered Linear Spaces, Maruzen, Tokyo, Japan, 1950.

[2] V. V. Chistyakov, "Modular metric spaces. I. Basic concepts," Nonlinear Analysis. Theory, Methods \& Applications A, vol. 72, no. 1, pp. 1-14, 2010.

[3] V. V. Chistyakov, "Modular metric spaces. II. Application to superposition operators," Nonlinear Analysis. Theory, Methods \& Applications A, vol. 72, no. 1, pp. 15-30, 2010.

[4] J. Musielak, Orlicz Spaces and Modular Spaces, vol. 1034 of Lecture Notes in Mathematics, Springer, Berlin, Germany, 1983.

[5] W. Orlicz, Collected Papers. Part I, II, PWN-Polish Scientific, Warsaw, Poland, 1988.

[6] M. A. Khamsi, W. M. Kozłowski, and S. Reich, "Fixed point theory in modular function spaces," Nonlinear Analysis. Theory, Methods \& Applications A, vol. 14, no. 11, pp. 935-953, 1990.

[7] M. A. Khamsi and W. M. Kozlowski, "On asymptotic pointwise contractions in modular function spaces," Nonlinear Analysis. Theory, Methods \& Applications A, vol. 73, no. 9, pp. 2957-2967, 2010.

[8] M. A. Khamsi and W. M. Kozlowski, "On asymptotic pointwise nonexpansive mappings in modular function spaces," Journal of Mathematical Analysis and Applications, vol. 380, no. 2, pp. 697-708, 2011.

[9] L. P. Belluce and W. A. Kirk, "Fixed-point theorems for families of contraction mappings," Pacific Journal of Mathematics, vol. 18, pp. 213-217, 1966.

[10] L. P. Belluce and W. A. Kirk, "Nonexpansive mappings and fixed-points in Banach spaces," Illinois Journal of Mathematics, vol. 11, pp. 474-479, 1967.

[11] F. E. Browder, "Nonexpansive nonlinear operators in a Banach space," Proceedings of the National Academy of Sciences of the United States of America, vol. 54, pp. 1041-1044, 1965.

[12] R. E. Bruck Jr., "A common fixed point theorem for a commuting family of nonexpansive mappings," Pacific Journal of Mathematics, vol. 53, pp. 59-71, 1974.

[13] R. DeMarr, "Common fixed points for commuting contraction mappings," Pacific Journal of Mathematics, vol. 13, pp. 1139-1141, 1963.

[14] T. C. Lim, "A fixed point theorem for families on nonexpansive mappings," Pacific Journal of Mathematics, vol. 53, pp. 487-493, 1974.

[15] K. Goebel and S. Reich, Uniform Convexity, Hyperbolic Geometry, and Nonexpansive Mappings, vol. 83 of Monographs and Textbooks in Pure and Applied Mathematics, Marcel Dekker, New York, NY, USA, 1984.

[16] K. Goebel, T. Sekowski, and A. Stachura, "Uniform convexity of the hyperbolic metric and fixed points of holomorphic mappings in the Hilbert ball," Nonlinear Analysis, vol. 4, no. 5, pp. 1011-1021, 1980.

[17] W. A. Kirk, "Fixed point theorems in $C A T(0)$ spaces and $\mathbb{R}$ trees," Fixed Point Theory and Applications, no. 4, pp. 309-316, 2004.

[18] W. A. Kirk, "Fixed points of asymptotic contractions," Journal of Mathematical Analysis and Applications, vol. 277, no. 2, pp. 645-650, 2003.

[19] W. A. Kirk, "Asymptotic pointwise contractions, plenary lecture," in Proceedings of the 8th International Conference on Fixed Point Theory and Its Applications, Chiang Mai University, July 2007.
[20] N. Hussain and M. A. Khamsi, "On asymptotic pointwise contractions in metric spaces," Nonlinear Analysis. Theory, Methods \& Applications A, vol. 71, no. 10, pp. 4423-4429, 2009.

[21] W. A. Kirk and H.-K. Xu, "Asymptotic pointwise contractions," Nonlinear Analysis. Theory, Methods \& Applications A, vol. 69, no. 12, pp. 4706-4712, 2008.

[22] M. A. Khamsi and W. A. Kirk, An Introduction to Metric Spaces and Fixed Point Theory, John Wiley, New York, NY, USA, 2001.

[23] W. M. Kozlowski, Modular Function Spaces, vol. 122 of Monographs and Textbooks in Pure and Applied Mathematics, Marcel Dekker, New York, NY, USA, 1988.

[24] W. M. Kozlowski, "Notes on modular function spaces I," Comment Mathematica, vol. 28, pp. 91-104, 1988.

[25] W. M. Kozlowski, "Notes on modular function spaces II," Comment Mathematica, vol. 28, pp. 105-120, 1988.

[26] D. W. Boyd and J. S. W. Wong, "On nonlinear contractions," Proceedings of the American Mathematical Society, vol. 20, pp. 458464, 1969. 


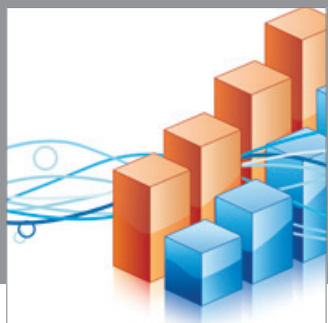

Advances in

Operations Research

mansans

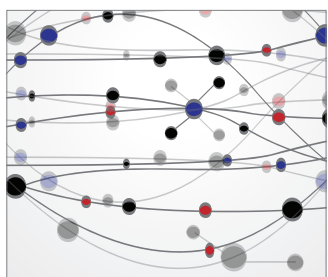

The Scientific World Journal
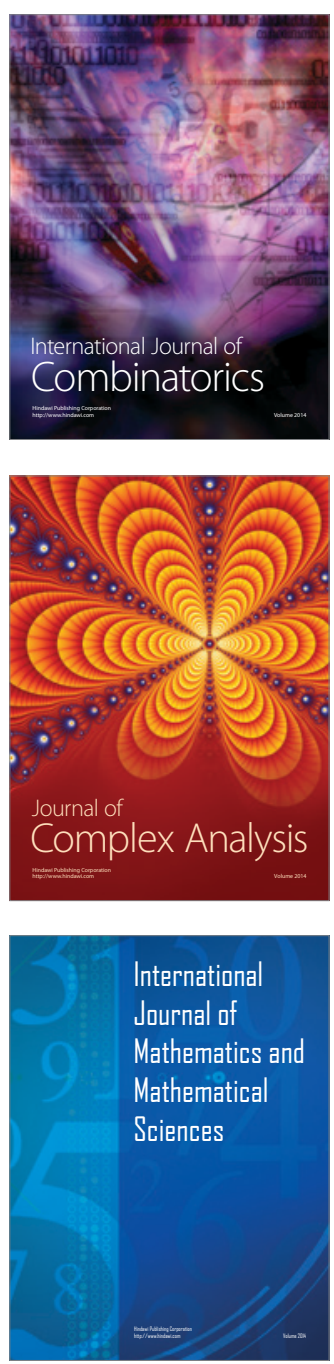
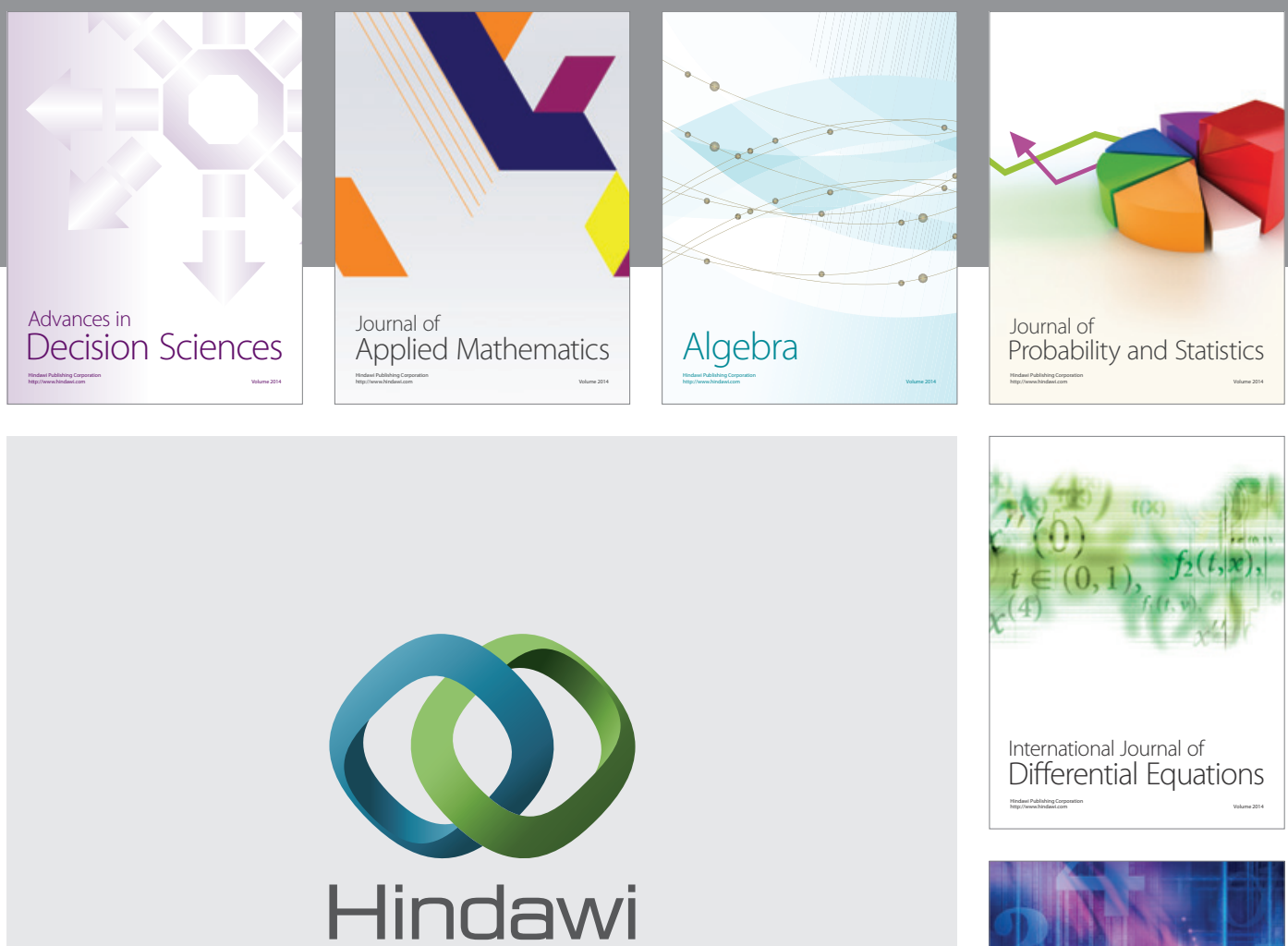

Submit your manuscripts at http://www.hindawi.com
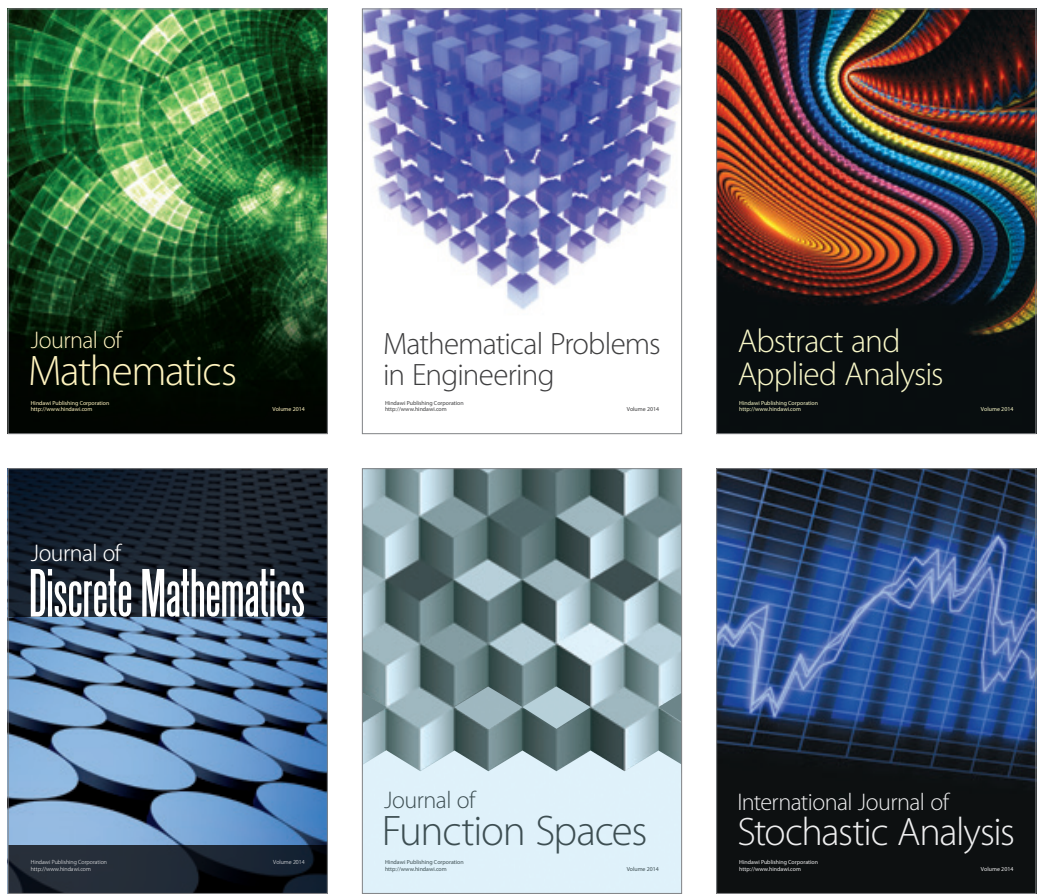

Journal of

Function Spaces

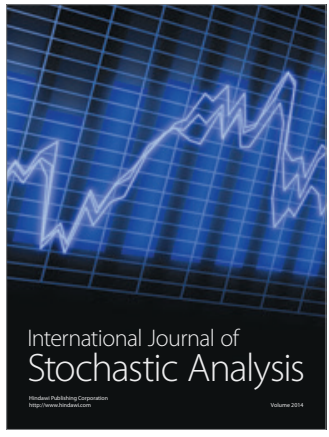

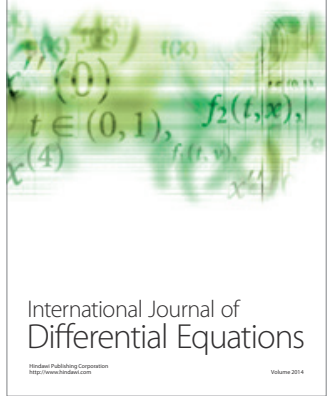
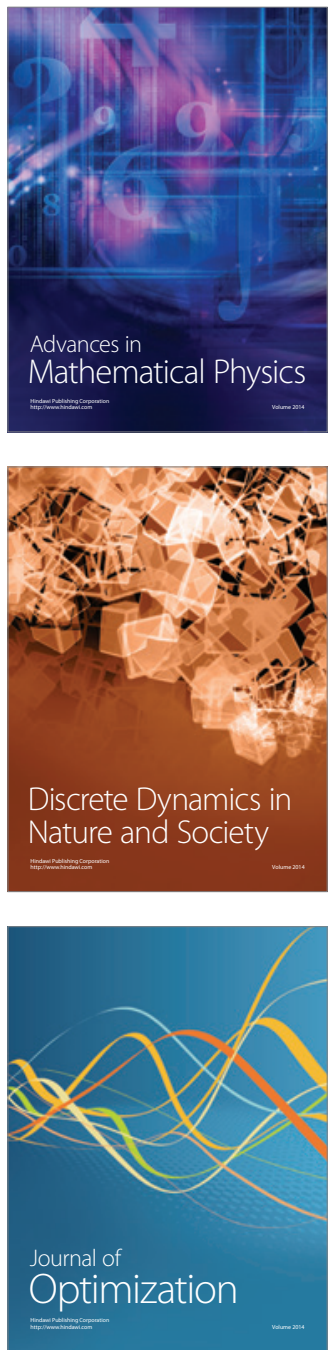